\title{
RELATOS DE EXPERIENCIA

\section{Educación ambiental para el desarrollo sostenible: enfoque desde San Luís, Santiago de Cuba}

Marcia Aparecida Silva Pimentel ${ }^{\mathrm{I} \text { II }}$

Marco Antonio Lopes San Jose ${ }^{\mathrm{III}}$, IV

Sandy Guillen Cerpav, vi

http://dx.doi.org/10.24109/2176-6681.rbep.100i255.4051

Universidade Federal do Pará (UFPA). Belém, Pará, Brasil. E-mail: <mapimentel@ufpa. br $>$; $<$ https://orcid.org/ 0000-0001-9893-9777>

II Doutora em Geografia pela Universidade de São Paulo (USP). São Paulo, São Paulo, Brasil.

III Universidad de Oriente (UO-Cuba). Santiago, Cuba. E-mail: <marco.lopez@ uo.edu.cu $>$; $<$ https://orcid. org/0000-0002 -2132-1482>

Iv Mestre em Biologia pela Universidad de Oriente (UO-Cuba). Santiago, Cuba.

v Universidad de Oriente (UO-Cuba). Santiago, Cuba E-mail: < sguillenc@ uo.edu.cu $>$; < https://orcid org/0000-0003 -2523-3671>.

vi Mestre em Química pela Universidad de Oriente (UO-Cuba). Santiago, Cuba.

\section{Resumen}

El presente trabajo propone tareas integradoras con enfoque interdisciplinario en el proceso de enseñanza-aprendizaje de la educación ambiental para el desarrollo sostenible. Las tareas se sustentan en fundamentos teóricos y prácticos, así como en el análisis de diferentes literaturas sobre el tema. Se aplica un diagnóstico para revelar las insuficiencias en la salida de esta línea metodológica, que constituye una prioridad en el trabajo metodológico y para la formación integral de los profesionales. A tal efecto, se diseñan ejercicios integradores para evaluar el nivel de generalización e integración de saberes para la resolución de tareas-problema, teniendo salida en diferentes asignaturas. Finalmente, se presentan resultados positivos en el desarrollo de habilidades investigativas, además de un mayor vínculo de los estudiantes con la comunidad y con las unidades docentes.

Palabras clave: educación ambiental; interdisciplinariedad; proceso de enseñanza-aprendizaje. 


\section{Resumo}

Educação ambiental para o desenvolvimento sustentável: abordagem de San Luís, Santiago de Cuba

Este trabalho propõe tarefas integradoras com abordagem interdisciplinar no processo de ensino-aprendizagem da educação ambiental para o desenvolvimento sustentável. As tarefas se baseiam em fundamentos teóricos e práticos, bem como na análise de diferentes literaturas sobre o assunto. Um diagnóstico é aplicado para revelar as insuficiências na saída dessa linha metodológica, que constitui prioridade no trabalho metodológico e na formação integral dos profissionais. São projetados exercícios de integração para avaliar o nível de generalização e integração de saberes na resolução de tarefas-problema, com saídas em diferentes disciplinas. Por fim, são apresentados resultados positivos no desenvolvimento de habilidades investigativas, além de um maior vínculo dos estudantes com a comunidade e com as unidades de ensino.

Palavras-chave: educação ambiental; interdisciplinaridade; processo de ensino-aprendizagem.

\section{Abstract \\ Environmental education for sustainable development: an approach of San Luis- Santiago de Cuba}

This work proposes integrative tasks with an interdisciplinary approach within the teaching - learning process for an environmental education for sustainable development. The tasks are based on theoretical and practical grounds, as well as on the analysis of different literatures on the subject. A diagnosis is run to reveal the inadequacies in the leaving of this methodological line, which is a priority for the methodological work and for the integral training of the professionals. Thus integrative exercises are developed to evaluate the level of generalizations and knowledge integration in the fulfilling of task-problems, wich may be applied to different subjects. Finally, positive results in the development of investigative skills are presented, as well as a stronger link between the students and the community and as well as teaching units.

Keywords: environmental education; interdisciplinarity; teachinglearning process. 


\section{Introducción}

El concepto de educación ambiental para el desarrollo sostenible está presente en un contexto de transformación de paradigmas del final del siglo pasado. Como afirma Leff (2002), los recientes acontecimientos en la historia "anuncian un cambio de época: el fin de los grandes proyectos de la modernidad y la emergencia de nuevos sentidos civilizatorios" (Leff, 2002, p. 5). De acuerdo con el autor, la sustentabilidad referente al desarrollo anuncia los límites de racionalidad económica y presenta otros valores relacionados con nuevas concepciones de vida, de justicia social y de responsabilidad con las futuras generaciones.

Por otra parte, las Conferencias Internacionales sobre el Medio Ambiente marcan las orientaciones de estudio e implantación de políticas educacionales sobre el tema. Desde la Conferencia sobre Educación Ambiental, realizada en Tbilisi, en 1977, inicia un amplio proceso a nivel global, orientado para crear las condiciones que forman una nueva conciencia sobre los valores de la naturaleza y para reorientar un producto de conocimiento basado en métodos de interdisciplinariedad y en los principios de la complejidad (Jacobi, 2003). Entre los años 1987 y 1992, las discusiones sobre las cuestiones ambientales culminaron con la construcción de la Agenda 21, documento firmado durante la Conferencia de las Naciones Unidas sobre el Medio Ambiente y Desarrollo, conocida como Rio 92. Para Munhoz (1991, p. 63), "la búsqueda de un modelo de desarrollo sostenible pasa necesariamente por la educación y por la implementación de políticas públicas de formación, información y concientización de la sociedad".

En Cuba, en el contexto de las transformaciones económicas, políticas, ideológicas y culturales generadas por la Revolución cubana durante la década de los 60 la educación superior se convirtió en un factor clave de las transformaciones sociales del país. Para Pérez Ones y Núñez Jover (2009), los programas de formación e investigación que las universidades cubanas fomentaron estaban orientados al desarrollo económico y social del país. Esa idea fue más fuerte después de la crisis del socialismo europeo de los años 80. Sin embargo, a pesar de esto, con apoyos financieros y políticos, las universidades crearon capacidades de producción y, en otros casos, establecieron relaciones muy estrechas con los sectores productivos, lo que resultó, por ejemplo, en las Oficinas de Transferencia de Resultados de Investigación (OTRI) (Pérez Ones; Núñez Jover, 2009).

El contexto internacional de los años 90 ya planteado influenció nuevos valores sobre la discusión del medioambiente, economía y sociedad. Para el gobierno cubano, se buscó las interacciones de la universidad con el resto de la sociedad, sobre todo para el desarrollo local. Desde el 2002, para llevar a cabo la universalización de la educación superior, fueron creadas las Sedes Universitarias Municipales (SUM), que han devenido después de un proceso de perfeccionamiento en las Filiales Universitarias Municipales (FUM) y, por último, en el año de 2010, fueron creados los Centros Universitarios Municipales (CUM), por el Acuerdo n. ${ }^{\circ} 6935$ del Comité Ejecutivo del Consejo de Ministros. Estas instituciones tienen un carácter integrador y 
deben actuar en el contexto del municipio como gestores de conocimiento y de innovación, para asegurar mayor racionalidad al desarrollo socioeconómico de cada municipio. Esta política pública vino a atender la universalización de la educación en relación con los estudios superiores y permitió certificar todos los conceptos de la responsabilidad social universitaria en Cuba. (Hernández, 2012; Hernández Gutiérrez; Pich Herrera; Benítez Cárdenas, 2013).

De esa manera, los CUM están relacionados con los proyectos de desarrollo local y son un ejemplo de la aplicación consecuente de los cambios fundamentales para la actualización del modelo económico cubano, como propone en los Lineamientos de la Política Económica y Social del Partido y la Revolución (PCC, 2011). Estos principios están relacionados con la comprensión de que el conocimiento es el tema central para la economía del siglo 21. Lage Dávila (2007) hizo esta afirmación en una intervención durante una mesa redonda sobre la Universidad para Todos. En esta ocasión, añadió que no se trata solamente del conocimiento científico en sentido tradicional, pero tiene importancia también lo que llamó de conocimiento tácito, que está basado en la experiencia de los trabajadores. En este contexto, los CUM tienen un protagonismo relevante en la creación de estrategias de desarrollo local, a través de la capacitación de profesionales de diferentes ramas de las ciencias para dar una respuesta integrada entre economía y problemas medioambientales locales.

El diagnóstico fáctico e integral al proceso formativo de profesores de Biología y Geografía, para secundaria básica del Centro Universitario de San Luis, de la Universidad de Oriente en Santiago de Cuba, tiene una duración de dos años para habilitarlos. Dicha habilitación requiere el desarrollo de habilidades integradoras y destreza en la solución de problemas profesionales, por ejemplo, los controles a clases, análisis de planes de clase, reuniones de carrera, asesorías y el análisis de los propios informes de evaluación de calidad. Se detectó actividades metodológicas con insuficiente carácter interdisciplinar para la orientación de ejercicios integradores en la carrera y, también, una torpe organización de la dinámica grupal en la solución de problemas. Existe, además, una incapacidad de concepción de tareas docentes para la planificación, orientación, ejecución y evaluación de ejercicios integradores con carácter interdisciplinar, debilidades en la propuesta integradora y rectora de la práctica sistemática como elemento fundamental del proceso formativo.

A tales efectos, se plantean las siguientes problemáticas: ¿De qué manera los profesores del equipo pedagógico pueden romper con la insuficiente preparación de los docentes del Centro Universitario Municipal de San Luís, de la Universidad de Oriente? ¿Cómo proponer tareas integradoras con carácter interdisciplinar en la dirección del proceso de enseñanza-aprendizaje de la educación ambiental para el desarrollo sostenible?

Ante esta situación, se traen los siguientes objetivos:

1. Presentar ejercicios integradores, con carácter interdisciplinar, para perfeccionar el proceso de enseñanza-aprendizaje de la educación 
ambiental para el desarrollo sostenible, teniendo el papel protagónico de los estudiantes según las transformaciones actuales de la educación superior cubana;

2. Exponer los principales resultados alcanzados en el proceso formativo integral de docentes pertenecientes al Curso de Nivel Medio Superior del Centro Universitario Municipal en el logro de la interdisciplinariedad, a través de la concreción práctica de un ejercicio integrador como base de la evaluación final de varias asignaturas del plan de estudio.

\section{Referentes teóricos}

Sobre educación ambiental para o desarrollo sostenible

Según Barbieri y Silva (2011, p. 53),

[. . . ] el debate sobre la educación ambiental para el desarrollo sostenible ha ganado popularidad a escala mundial desde la última década del siglo pasado y viene encontrando un amplio espacio, a medida que las crisis sociales y ambientales de dimensión planetaria continúan amenazando el futuro de la humanidad y del propio planeta.

Jacobi (2003) ya afirmaba que es cada vez más favorable pensar en la relación entre medioambiente y educación para la ciudadanía, lo que exige el conocimiento sobre procesos sociales cada vez más complejos, como la comprensión de los riesgos ambientales progresivamente más intensos. Lo mismo piensa Reigota (2017) cuando afirma la educación ambiental como educación política, a partir del análisis de las relaciones políticas, económicas, sociales y culturales entre la humanidad y la naturaleza, y las relaciones entre los seres humanos para superar los mecanismos de dominación que impiden la democracia. Carvalho (2017) también está de acuerdo con la visión socioambiental e interdisciplinar que piensa la naturaleza como un campo de interacción entre la cultura, la sociedad y la base física. Eso significa, por tanto, un avanzo en el concepto de medioambiente y de educación ambiental.

\section{Sobre la interdisciplinariedad y la formación de profesores}

La interdisciplinariedad y las prácticas educativas integradoras tienen sus bases en la internacionalización y en la complejización de la vida social, económica, política y cultural, cuyo principal motor es el desarrollo de la ciencia y de la tecnología. Para Núñez Jover y Alcazar Quiñones (2016, p. 197),

[. . . ] el concepto de "ciencia integrada" surge por la presión creciente por una ciencia relevante y creíble, capaz de informar a las políticas públicas, que influya en la calidad de vida de las personas. Está planteado un reclamo a profesionales e investigadores de asumir problemas del mundo real y colaborar en las transformaciones. 
En otras palabras, significa un cambio de actitud frente a los problemas del conocimiento, una sustitución de la concepción fragmentaria por una unitaria del hombre y la realidad en que vive y, como afirma Fazenda (1993), la interdisciplinariedad presupone un compromiso con la totalidad. En la literatura sobre el tema existe consenso en destacarla como una forma de pensar y de proceder para conocer y resolver cualquier problema de la realidad que requiera de la convicción y de la cooperación entre las personas. Para Ander-Egg (1994), abordar la interdisciplinariedad en el ámbito educativo significa considerar cualquier intervención que se realice como un aspecto de la totalidad o de las totalidades de las que forma parte.

Esta precisión es clave para entender el carácter sistémico de la interdisciplinariedad, fundamento para la elaboración de una estrategia de enseñanza-aprendizaje, partiendo de entender el carácter de sistema complejo de este proceso. En la formación de docentes es necesario que esté presente el principio interdisciplinar-profesional, que es aquel que dirige el proceso de enseñanza-aprendizaje hacia la preparación de un futuro profesional capaz de realizar transferencias de contenidos que le permiten solucionar, holísticamente, los problemas que enfrentará en su futuro desempeño profesional.

La interdisciplinariedad es una concepción integradora de trabajo del proceso docente educativo que refleja la creciente interpretación del saber como reflejo de la realidad natural y social. En su esencia, la interdisciplinariedad tiene como elementos clave el conocimiento profundo de las disciplinas, la actividad de las personas que la desarrollan, su cooperación, flexibilidad de análisis, comunicación, enriquecimiento mutuo de conocimientos, integración al grupo en la formación de valores, así como la profundización y solución creativa de los problemas. Sobre interdisciplinariedad, Leff (2011) argumenta que implica en un proceso de interrelación de procesos, conocimientos y prácticas que desborda y trasciende el campo de la investigación y de la enseñanza, en lo que se refiere estrictamente a las disciplinas científicas y a sus posibles articulaciones.

En las escuelas, la interdisciplinariedad ha sido tratada en ocasiones como una cuestión teórica. Por lo tanto, se hace necesario concretarla en la práctica pedagógica, mediante acciones específicas, para lo cual tienen un papel esencial los departamentos docentes. Concebidos como la célula fundamental del trabajo político y pedagógico en la escuela, los departamentos son elementos aglutinadores y coordinadores entre las asignaturas, son encargados, por tanto, de potenciar la interdisciplinariedad.

La educación ambiental en los planes de formación de docentes se logra a partir del trabajo metodológico de los colectivos de asignaturas ${ }^{1}$ y constituye un aspecto esencial para lograr la efectividad de la valoración interdisciplinaria, de acuerdo con los objetivos definidos. Esto implica que, en tal contexto, la integración de contenidos se desarrolla con una estrecha coordinación entre cada una de ellas y la adecuada definición de los conceptos y habilidades de acuerdo con los siguientes pasos:
Materias que se enseñan en un curso y que forman parte de un programa de estudios. 


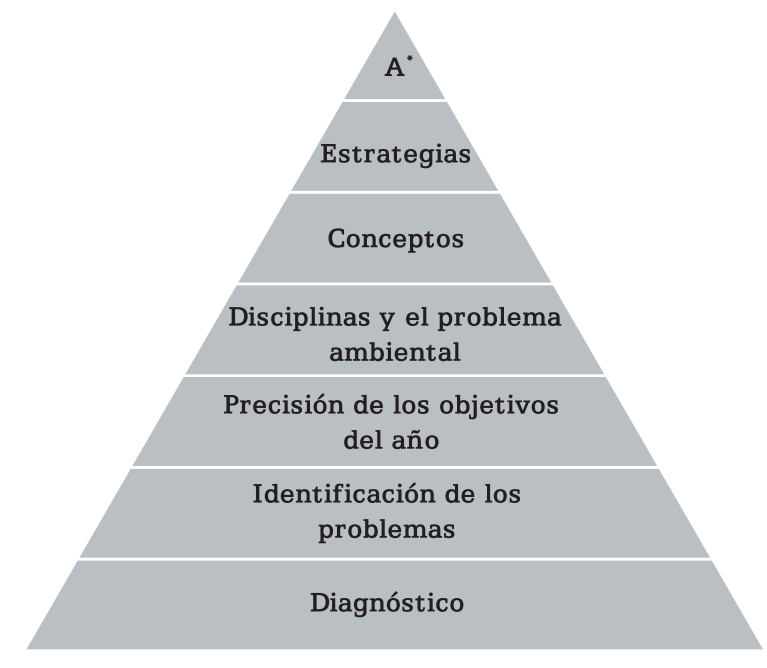

Figura 1 - Organización del proceso de enseñanza interdisciplinar

Fuente: elaborada por los autores.

Nota: *A: evaluación.

Todo esto implica un proceso de maduración y conceptualización de la problemática ambiental que abarca aspectos de índole educativos, científico-técnicos, sociales, morales, culturales y éticos. El trabajo en esta esfera queda con una organización que favorece la integración desde diferentes áreas y se forma conciencia de la pluralidad de acciones para dar solución a un problema.

Una de las vías por las que se pretende utilizar la práctica interdisciplinar en nuestro contexto son las llamadas "tareas integradoras". Dichas tareas son introducidas en un proceso de enseñanza-aprendizaje que, en la práctica, no ha experimentado cambios del referido proceso sin que exista un enfoque integrador desde un currículo aún fragmentado, que se materialice en procederes pedagógicos concretos que proporcionen a los alumnos herramientas para la integración de los contenidos.

La interdisciplinariedad es reflejo y concreción de la compleja realidad en toda actividad humana dirigida realmente a conocerla, comprenderla y transformarla.

Al ser la interdisciplinariedad principio estructurante del currículo, a la vez que proceso, en el ámbito educativo, no puede ser resultado de la actividad espontánea, aislada y ocasional, sino una de las bases de una concepción pedagógica centrada en el sujeto, meditada, instrumentada y ejecutada, con enfoque sistémico por el colectivo pedagógico. La intervención del colectivo no debe limitarse a la relación entre los conocimientos sin abarcar toda la labor educativa, basada en la propia actuación profesional, en la motivación y en el ejemplo de los profesores y directivos.

Para Perera Cumerma (2000, p. 48),

[. . .] no es solo una cuestión teórica, académica, sino ante todo una práctica, es un proceso vinculado, en su esencia, con una determinada 
forma de pensar y de actuar de las personas para conocer y resolver cualquier problema de la realidad y requiere de su convicción, actitud de cooperación, cultura y flexibilidad, por lo que no es una moda ni un esquema que pueda imponerse.

Es criterio de los autores que la interdisciplinariedad no se refiera a simples relaciones entre disciplinas o asignaturas, sino a interrelaciones que generan síntesis, que parten de la existencia de sistemas complejos que originan una forma de organización científica de trabajo integrado, donde el conocimiento se mueve dialécticamente hasta alcanzar las más amplias generalizaciones.

Derivándose de estas relaciones, se determinan dos funciones básicas que están implícitas en las relaciones interdisciplinarias: interrelación y cooperación. Para Salazar Fernández (2001), la interdisciplinariedad presupone una forma de explicar la realidad educativa en su conjunto, con apoyo en el establecimiento de nexos e interrelaciones entre las disciplinas, capaces de generar nuevas síntesis y de superar el reduccionismo en el estudio y solución de los problemas de la práctica educativa. No solo a través del establecimiento de nexos interdisciplinarios, sino entre los sujetos que la practican.

Sobre los ejercicios integradores interdisciplinarios, Martínez Rubio (2003) los define como siendo situaciones de enseñanzaaprendizaje estructuradas a partir de un eje integrador (problema profesional) conformadas esencialmente por problemas y tareas docentes interdisciplinarias. Su finalidad es enseñar a los estudiantes a relacionar los saberes especializados, apropiados desde la disciplinaridad, mediante la conjugación de métodos de investigación científica y didáctica, y la articulación de las formas de organización del componente académico con las del investigativo-laboral. Su resultado es la formación de saberes integrados expresados en nuevas síntesis y en ideas cada vez más totales de los objetos, fenómenos y procesos de la práctica educativa, en consecuencia de comportamientos y valores inherentes a su profesión.

El problema docente interdisciplinario presupone el planteamiento o identificación de contradicciones que se manifiestan en el objeto de trabajo del profesional y que, llevadas a situaciones de enseñanza-aprendizaje, motivan la búsqueda y estimulan el establecimiento de nexos y de relaciones interdisciplinarias para su solución.

Por tarea docente interdisciplinaria se entiende el conjunto de acciones o interrogantes que organizan la búsqueda de solución a los problemas, mediante el establecimiento de nexos entre los contenidos de diferentes disciplinas y la conjugación de diversos métodos y formas de organización del currículo y de las relaciones entre los sujetos del proceso.

Para Perera Cumerma (2000), las tareas integradoras son aquellas cuya solución requiere una real integración de los contenidos, su aplicación y generalización. Ellas no deben cumplir la mera función de evaluación de los contenidos, sino deben concebirse como momentos culminantes, hitos del proceso de enseñanza-aprendizaje, que contribuyen a valorar tanto el desarrollo integral de cada estudiante como del propio proceso. 
Estas actividades no son aisladas, sino que forman parte de un sistema de actividades interdisciplinarias. Mediante la realización de este sistema se va revelando a los estudiantes la relación existente entre distintos fenómenos o procesos de la realidad, aparentemente inconexos. Es para eso que resulta esencial el conocimiento de los conceptos, métodos y leyes científicas, unido a la posibilidad creciente y paulatina de integrarlos y aplicarlos, partiendo de una actitud crítica y reflexiva ante los problemas que se les planteen. Los estudiantes van desarrollando habilidades para realizar operaciones de análisis y de síntesis y de transferencias de contenidos.

Las diferentes asignaturas para abordar la educación ambiental para el desarrollo sostenible como eje transversal deben identificar problemas ambientales locales o presentes en la localidad escolar y dar cumplimiento al objetivo planteado. Además, debe explicar las causas y efectos de estos problemas que afectan, así como su incidencia en el cambio climático para proponer acciones encaminadas a su disminución o solución con la participación de las instituciones educativas, de las familias y de la comunidad con sus representantes. De esta forma, se logra una enseñanza significativa y prepara a los estudiantes como entes sociales transformadores que se habilitan para dirigir el proceso docente educativo en el nivel de secundaria básica.

\section{Procedimientos metodológicos}

\section{Área de estudio}

San Luis (de las Enramadas) es un municipio de la provincia de Santiago de Cuba, de tamaño mediano desde el punto de vista geográfico, a solo veintiocho kilómetros $\left(28 \mathrm{~km}^{2}\right)$ de la capital Santiago de Cuba.

El territorio tiene una extensión de $764.4 \mathrm{Km}^{2}$ y su población asciende a 82454 habitantes, concentrándose mayor cantidad en la zona urbana (46 461) que en la zona rural (35993), distribuidos en 17 consejos populares. Es el cuarto mayor municipio por su extensión superficial y el quinto por su población, en la provincia Santiago de Cuba. Los principales asentamientos poblacionales son San Luis (cabecera municipal), Chile y Dos Caminos, situados en el eje del Ferrocarril Central de Cuba. Además, San Luis posee otros 63 asentamientos rurales.

El municipio San Luis no está exento de problemas medioambientales que afectan al mundo. En este sentido, no se logra disminuir el efecto de degradación de los suelos. No se ha logrado implementar de forma generalizada la agricultura sostenible para garantizar la alimentación. Además, existen insuficiencias en el manejo forestal sostenible, en determinados momentos del año, por sequías que producen incendios forestales, lo que influye en la pérdida de la biodiversidad. También se aprecian manifestaciones en zonas estatales y urbanas de vertimiento inadecuado de residuos líquidos y sólidos, sin un accionar científico que permita su reúso y tratamiento. No se ha controlado la contaminación como 
resultado del manejo inadecuado de productos sólidos, existe contaminación en las zonas urbanas, producto del efecto de invernadero por gases, tales como CO2, CH4, N20, HFC, PFC, entre otros. Esos problemas influyen en el estado de salud de los habitantes y dificultan una adecuada higiene e integración ecológica.

El renglón económico fundamental del municipio es la producción de azúcar, que representa el 60 \% de la producción mercantil y el mayor fondo exportable. Existe actualmente una industria dedicada a la producción azucarera, el Complejo Agroindustrial Paquito Rosales, que acumula un monto de 79 millones de caña molible, con 14 centros de acopios y 72 combinadas. Esto significa que los complejos agroindustriales han mantenido un plan de producción estable.

En importancia económica, le sigue la producción cafetalera en la cual se aprecia un rendimiento de 83 \% en el año 1992 con relación al año 1977. El café es un producto bien dado en el territorio, gracias a un relieve montañoso donde se siembra en el suelo con sombra, principalmente en zonas montañosas. El suelo se caracteriza por ser generalmente ferralítico rojo.

Cabe destacar, además, que también se produce el tabaco manufacturado, conocido como habano, que es vendido a un alto costo mundialmente; en el territorio se cuenta con una fábrica para la exportación e importación de este producto.

\section{Procedimientos}

Este ejercicio integrador se orienta en la primera semana del semestre para el logro de momentos culminantes al finalizarlo, en función de detectar los hitos del proceso de enseñanza-aprendizaje, que contribuyen a valorar tanto el desarrollo integral de cada estudiante como del propio proceso, aunque se explica a los estudiantes que será tomado para evaluar en taller final asignaturas que no tienen prueba final. Lo realizarán por equipos, según estén organizados, para realizar la práctica sistemática en la secundaria básica.

Los estudiantes realizarán un informe para entregar una semana antes de la última semana de docencia; este debe ser manuscrito y con un mínimo de 10 cuartillas y un máximo de 15. El informe constará de introducción, desarrollo, conclusión, bibliografía y anexos. Cada equipo presentará los resultados en diapositivas. Para esto, cuentan con 10 minutos y luego se realiza el taller, se evaluarán por equipo e individualmente, según desempeño en la exposición y en el taller. Se evaluará en base a 100 puntos.

Se le orientan los parámetros a evaluar: ajuste al tema, actualización, claridad en las ideas, calidad del informe escrito, ortografía, limpieza, estética, calidad de la presentación, ajuste al tiempo, nivel de independencia, expresión oral, creatividad, calidad de las intervenciones y habilidades en el uso de la informática. Al finalizar el taller, se realiza la autoevaluación, autovaloración y la coevaluación, significando los aspectos positivos y recomendaciones. 
Dentro de las acciones a realizar para cumplimentar el objetivo planteado, se les indica a los estudiantes:

1. Realizar un mapa que represente la comunidad escolar y mencionar las transformaciones medioambientales ocurridas en la localidad, señalarlas y nombrarlas en el mapa elaborado;

2. Hacer alusión a acciones que se desarrollan contra la contaminación ambiental, mencionar los principales problemas ambientales e introducir una propuesta de acciones educativas de mejoramiento y conservación medioambiental;

3. Elaborar un cartel o afiche que exprese algunas acciones a favor de la protección del medioambiente y ponerlo en un lugar visible en su localidad;

4. Participar, junto a los alumnos del grupo de práctica laboral y con los miembros de la comunidad, en el saneamiento de un área afectada;

5. Mencionar logros de la institución educativa como centro cultural más importante de la comunidad.

\section{Resultados y discusión}

Se obtienen resultados positivos. Los estudiantes se ajustaron a las orientaciones y realizaron informes de calidad, demostrando creatividad en las propuestas de mejoramiento ambiental con la participación de miembros de la comunidad. Además, expusieron de forma clara aspectos didácticos y metodológicos al realizar un estudio en las asignaturas de Biología y Geografía de cómo dar salida al eje transversal de la educación ambiental, a través de clases y actividades extradocentes y extraescolares. En la presentación y discusión de los resultados, mostraron independencia, claridad en las ideas y dominio del contenido y creatividad, así como dominio de las Técnicas de la Informática y de las Comunicaciones.

De forma general, mostraron habilidades investigativas para buscar la información, mayor vínculo con la comunidad escolar y con las propias unidades docentes donde realizan las prácticas. En este sentido, se logra mayor protagonismo estudiantil en procesos sociales y de su propia formación integral. Eso colabora con la idea de Santos y Jacobi (2017) de que los ambientes pedagógicos que defienden prácticas y actitud ambiental son un camino para una sociedad sostenible.

Esas prácticas llevaron al desarrollo de habilidades comunicativas tanto orales como escritas, los informes muestran la calidad requerida y se ajustan a los parámetros establecidos y a la búsqueda de información y presentación de las diapositivas. El 100 \% de los estudiantes se presentaron al encuentro de evaluación en el que nueve estudiantes obtuvieron la máxima calificación, de 100 puntos, y siete con notas en el rango entre 90 y 99 puntos. Se observan resultados positivos en el logro de la motivación, el trabajo en equipo, la responsabilidad, la búsqueda de información en soporte digital, el colectivismo y la optimización del tiempo. 
Los estudiantes expresaron satisfacción por la forma en que se presentaron los resultados y por las calificaciones recibidas, el desarrollo de habilidades investigativas, además de un mayor vínculo con la comunidad y con las unidades docentes. Señalaron como obstáculos superados las dificultades en la búsqueda de información digital en internet y, en otra dirección, en el trabajo de integración de los miembros de la comunidad a las actividades.

Los directivos de las unidades docentes coinciden en que los estudiantes se mostraron responsables, contribuyeron a elevar a planos superiores el protagonismo de la institución educativa en su rol de centro cultural más importante de la comunidad, a la vez que se contribuyó a perfeccionar el trabajo metodológico y la calidad de las clases como vía fundamental para instruir y educar la personalidad, lo que se demostró en los resultados de los controles a clases con un $100 \%$ de clases bien evaluadas.

En el acto de presentación y discusión de los resultados, participaron comunitarios invitados y familiares que habían colaborado con los estudiantes durante la realización de las actividades. Expresaron haber aprendido sobre la problemática ambiental local y estar en mejores condiciones para cuidar el medioambiente con acciones responsables que garanticen calidad de vida. También manifestaron agradecimiento al colectivo de docentes por la labor formativa realizada con los estudiantes, a la que calificaron de excelencia por rasgos de la personalidad que exhiben los graduados en su actuar diario en el ámbito familiar y comunitario.

Los directivos del Centro Universitario Municipal que observaron el taller final coincidieron en que los estudiantes se prepararon eficientemente, mostrando habilidades profesionales y comunicativas, así como un trabajo de excelencia en la caracterización ambiental de la localidad que debe servir de referente a posteriores estudios que se realicen.

\section{Consideraciones finales}

Existe un amplio marco teórico conceptual relacionado con la interdisciplinariedad en función de la educación ambiental para el desarrollo sostenible como eje transversal.

La realización de ejercicios integradores es una etapa importante y no un producto acabado de la interdisciplinariedad. Los ejercicios integradores interdisciplinarios como situaciones de enseñanza-aprendizaje estructuradas a partir de un eje integrador (problema profesional), conformadas esencialmente por problemas y tareas docentes interdisciplinarias, tienen finalidad de enseñar a los estudiantes a relacionar los saberes especializados apropiados. Partiendo desde los diferentes campos de conocimientos, mediante la conjugación de métodos de investigación científico y didácticos, y la articulación de las formas de organización del componente académico con las del investigativo-laboral. Su resultado es la formación con saberes integrados, expresados en nuevas síntesis y en ideas cada vez más totales de los objetos, fenómenos y procesos de la práctica educativa, en consecuencia de comportamientos y valores inherentes a su profesión. 
La interdisciplinariedad es un proceso y una filosofía de trabajo, es una forma de pensar y de proceder para conocer la complejidad de la realidad objetiva y resolver cualquiera de los complejos problemas que esta plantea.

La interdisciplinariedad facilita el aprendizaje de los estudiantes, quienes reciben los conocimientos debidamente articulados, a la vez que revela el nexo entre los distintos fenómenos y procesos de la realidad que son objeto de estudio, superando la fragmentación del saber. Capacita a los alumnos para hacer transferencias de contenidos y aplicarlos en la solución de problemas nuevos. Implica formar en los estudiantes valores, actitudes y una visión del mundo globalizadora.

\section{Referencias}

ANDER-EGG, E. Interdisciplinariedad en educación. Buenos Aires: Editorial Magisterio del Río de la Plata, 1994.

BARBIERI, J. C.; SILVA, D. Desenvolvimento sustentável e educação ambiental: uma trajetória comum com muitos desafíos. RAM: Revista de Administração Mackenzie, São Paulo, v. 12, n. especial 3, p. 51-82, maio/ jun. 2011.

CARVALHO, I. C. M. Educação ambiental e a formação do sujeito ecológico. 6. ed. São Paulo: Cortez, 2017. (Coleção Docência em Formação).

CASTRO RUZ, F. El diálogo de civilizaciones. La Habana: Oficina de Publicaciones del Consejo de Estado, 2007.

FAZENDA, I. C. A. (Org.). Prácticas interdisciplinares na escola. Cortez: São Paulo, 1993.

HERNÁNDEZ GUTIÉRREZ, D. N.; PICH HERRERA, B.; BENÍTEZ CÁRDENAS, F. Los Centros Universitarios Municipales: un estudio de caso de responsabilidad social universitaria en Cuba. Revista Congreso Universidad, [La Habana], v. 2, n. 2, p. 1-13, 2013. Disponible en: $<$ http://www.congresouniversidad.cu/revista/index.php/rcu/article/ view/494/459>. Acceso en: 30 abr. 2019.

HERNÁNDEZ, C. A. Roles de los Centros Universitarios Municipales (CUM) en el desarrollo local de los municipios cubanos. Revista de Ingenierías USBMed, Medellín, v. 3, n. 1, p. 43-49, enero/jun. 2012.

JACOBI, P. Educação ambiental, cidadania e sustentabilidade. Cadernos de Pesquisa, São Paulo, n. 118, p. 189-205, mar. 2003. 
LAGE DÁVILA, A. Cuba ha creado las bases para el tránsito a una economía basada en el conocimiento. La Habana, 2007. Disponible en: $<$ http://www.cubadebate.cu/opinion/2007/10/05/cuba-ha-creado-lasbases-para-el-transito-a-una-economia-basada-en-el-conocimiento/ $>$. Acceso en: 3 mayo 2019.

LEFF, E. Complexidade, interdisciplinaridade e saber ambiental. Olhar de Professor, Ponta Grossa, v. 14, n. 2, p. 309-335, 2011.

LEFF, E. Saber ambiental: sustentabilidad, racionalidad, complejidad, poder. Buenos Aires: PNUMA; Centro de Investigaciones Interdisciplinarias en Ciencias y Humanidades/UNAM; Siglo Ventiuno Editores, 2002.

MARTINEZ RUBIO, B. N. La interdisciplinariedad en la ciencia, la didáctica y el currículo. Lima: Fondo Editorial del Pedagógico San Marcos, 2003.

MUNHOZ, T. Desenvolvimento sustentável e educação ambiental. Em Aberto, Brasília, v. 10, n. 49, p. 63-64, jan./mar. 1991.

NUÑEZ JOVER, J.; ALCAZAR QUIÑNONES, A. ¿Universidad y desarrollo local/territorial? Argumentos conceptuales y sugerencias para las políticas institucionales. In: NUÑEZ JOVER, J.; ALCAZAR QUIÑNONES, A. (Coord.). Universidad y desarrollo local: contribuciones latinoamericanas. La Habana: Editorial Universitaria Félix Varela/Unión de Universidades de América Latina y el Caribe (UDUAL), 2016. p. 191-204.

PARTIDO COMUNISTA DE CUBA (PCC). Lineamientos de la política económica y social del partido y la revolución. La Habana, 2011.

PERERA CUMERMA, L. F. La formación interdisciplinaria de los profesores de ciencias: un ejemplo de la enseñanza aprendizaje de la Física. 2000. 142 h. Tesis (Doctorado en Ciencias Pedagógicas) - Departamento de Física, Facultad de Ciencias, Instituto Superior Pedagógico "Enrique José Varona", La Habana, 2000.

PÉREZ ONES, I.; NÚÑ̃EZ JOVER, J. Higher education and socio-economic development in Cuba: high rewards of a risky high-tech strategy. Science and Public Policy, Londres, v. 36, n. 2, p. 97-101, Mar. 2009.

REIGOTA, M. O que é educação ambiental? São Paulo: Hedra, 2017.

SALAZAR FERNÁNDEZ, D. La formación interdisciplinaria del futuro profesor de Biología en la actividad científico investigativa. 2001. $111 \mathrm{~h}$. Tesis (Doctorado en Ciencias Pedagógicas) - Instituto Superior Pedagógico "Enrique José Varona", La Habana, 2000. 
SANTOS, V. M. N.; JACOBI, P. R. Educação, ambiente e aprendizagem social: metodologias participativas para geoconservação e sustentabilidade. Revista Brasileira de Estudos Pedagógicos, Brasília, v. 98, n. 249, p. 522-539, maio/ago. 2017.

Recebido em 13 de julho de 2018.

Aprovado em 8 de maio de 2019. 
